Published in final edited form as:

J Am Dent Assoc. 2010 March ; 141(3): 279-284.

\title{
Hyposalivation and xerostomia in dentate older adults
}

\author{
R. Constance Wiener, DMD, Bei Wu, PhD, Richard Crout, DMD, PhD, MS, Michael Wiener, \\ DMD, Brenda Plassman, PhD, Elizabeth Kao, DMD, and Daniel McNeil, PhD
}

\section{Abstract}

Background-Older adults are susceptible to reduced saliva production related to certain medications, radiation and chronic conditions. Many of these people have many physical and oral health problems and limited access to dental care. The use of effective screening tools for xerostomia and hyposalivation would be helpful in identifying those at risk. The authors conducted a study to investigate the association between three measures of oral dryness: hyposalivation (low unstimulated salivary flow), self-reported xerostomia and clinically assessed dry mouth.

Methods-The authors included a convenience sample of 252 nondemented and dentate West Virginia participants 70 years and older who were part of a larger study on oral health and cognition among older adults. Participants completed a self-reported xerostomia index, provided an unstimulated salivary sample and underwent an oral assessment for the study.

Results-Twenty-eight (11.1 percent) had hyposalivation, eight of whom reported having xerostomia (sensitivity $=28.6$ percent). Of the 43 participants who reported having xerostomia, only eight had hyposalivation (positive predictive value $=18.6$ percent). Hyposalivation and self-reported xerostomia were not significantly related. Clinically assessed dry mouth correlated modestly, but significantly, with hyposalivation and self-reported xerostomia.

Conclusions-Obtaining routine unstimulated salivary flow rates in addition to self-reported information and oral evaluations may increase early detection of oral dryness, which would assist in implementing early interventions to improve patients' quality of life.

Clinical Implications-Visually inspecting oral tissues for dryness and asking a patient if his or her mouth is dry are insufficient measures for clinicians to use to determine if the patient has hyposalivation. The authors recommend that clinicians determine the patients' unstimulated salivary flow rate.

\section{Keywords}

Xerostomia; salivary flow; geriatrics

Saliva plays a crucial role in oral health. It buffers acids, has antibodies, helps prevent gingival mucosal erosions and ulcerations, and aids in tooth remineralization. When salivary function is diminished, there is more risk of patients developing caries, experiencing denture discomfort, and having diseases such as candidiasis than there is in patients who have normal salivary flow rates. ${ }^{1,2,3}$ Xerostomia is oral dryness. ${ }^{4}$. Medications with antisialogogic effects are the most frequent causes of xerostomia. These include anticholinergic, antidepressant, antipsychotic, diuretic, antihypertensive, sedative and anxiolytic, antihistamine, opiod analgesic agents as well as nonsteroidal anti-inflammatory drugs. $1,4,5,6$ Some biological causes of xerostomia are having history of radiation to the head and neck, diseases of the salivary gland, diabetes, alcoholic cirrhosis, cystic fibrosis, hormonal imbalance, auto-immune diseases (such as Sjogren's syndrome, rheumatoid arthritis, systemic lupus erythematosus), and other diseases. $4,5,6$ Social and psychological factors, such as depression, anxiety, and stress are also causes. $4{ }^{5} .6$ 
Xerostomia is a common problem. In one study, the author found that $46 \%$ of the participants had xerostomia. 6 The authors of a review article found that between 17 and $29 \%$ of older adults had xerostomia, with a higher prevalence in women. ${ }^{1}$ Xerostomia often occurs with other oral conditions such as idiopathic dysesthesia (stomatalgia or "burning mouth syndrome"), parageusia, dysphagia, halitosis, caries, and periodontal disease. ${ }^{2}, 3,6$ Xerostomia may hinder speaking and the use of prosthetics and may be a factor in malnutrition. $2^{2,3,6}$ The psycho-social aspects of xerostomia may range from a mild effect on self-rated oral health, to frustration, embarrassment, unhappiness, or substantial disruptions in daily living. 3,7

Salivary gland hypofuntion or hyposalivation is the condition of having reduced saliva production which is different from xerostomia. . The best way to diagnose hyposalivation is to measure the salivary flow. Salivary flow rates have been used as the basis for diagnosing hyposalivation in a large scale study. ${ }^{8}$ The average unstimulated whole salivary flow rate is $0.3-0.4 \mathrm{ml} /$ minute during waking hours ${ }^{9}$ An unstimulated rate of $0.1 \mathrm{ml} / \mathrm{minute}$ or less indicates hyposalivation. ${ }^{9,10}$ People with hyposalivation experience inadequate bicarbonate and urea buffering, remineralization, and sugar and acid clearance and may result in an increased caries rate. $1,9,11$

People 65 years and older are most at risk of developing xerostomia and hyposalivation. In a three year study, researchers found that older adults with low salivary flow rates were more likely to lose at least one tooth than were older adults with normal salivary flow rates ${ }^{11}$. Many older adults have poor health, and chronic health conditions, and they neglect their dental care. ${ }^{12}$ Health care use often is based on the threshold of awareness with health status. ${ }^{13}$ If a person's self awareness of physical symptoms is blunted, additional support and health screenings may be helpful to identify medical and oral health problems.

Oral tissue dryness is complex. An person may experience xerostomia with or without hyposalivation; experience hyposalivation with or without xerostomia or have average salivary flow and normal sensation. ${ }^{14}$ Clinical assessment of oral dryness is another important component of care. ${ }^{10} \mathrm{An}$ individual may have clinically identified oral dryness with or without xerostomia and with or without hyposalivation. We hypothesized that older adults' unstimulated salivary flow rates are discrete from their self-reports of having xerostomia and from the visual assessment of a dry mouth, and that the results from self-reports of having xerostomia, visual assessment, and unstimulated salivary flow rates all are needed to identify oral dryness. We conducted a study to investigate the association between three measures of oral dryness: hyposalivation, selfreported xerostomia, and dry mouth identified by means of clinical assessment.

\section{PARTICIPANTS AND METHODS}

The participants were part of a larger study on oral health and cognition among older adults in West Virginia. The West Virginia University Institutional Review Board approved all of the procedures, and we obtained consent from all participants. We provided participants with gift cards to a local retailer as compensation for participating in the study We collected data from Aug. 1, 2007-April 30, 2009. The sample was a convenience sample of 269 residents of West Virginia who were at least 70 years old and had 4 or more natural teeth. We excluded 17 participants who had received the diagnosis with dementia because of concerns about the reliability of self-reported oral health information per our study protocol. The final sample included 252 people who were recruited from various locations across West Virginia by means of multiple strategies described previously. ${ }^{15}$

We conducted an oral assessment with all participants. This assessment included three measures of oral dryness--self-reported responses on the Xerostomia Inventory (XI), clinically 
assessed dry mouth, and measured whole unstimulated salivary flow. Trained research assistants administered an eleven-item, Likert-style XI. ${ }^{14}$ Each item in the inventory had a scoring range of 1 to 5, corresponding with "never," "hardly ever," "occasionally," "fairly often," and "very often," respectively. We summed the participants' responses to the items to determine the XI score. We characterized people who responded "fairly often," or "very often" as having positive responses. The XI included the item "My mouth feels dry"; we defined people who responded positively to it as having reported having xerostomia.

A dentist, (R.C.W., R.C., M.W., or E.K.) or a dental hygienist clinically assessed hyposalivation and measured whole unstimulated salivary flow. We used only the unstimulated salivary flow rate, as opposed to the stimulated salivary flow rate, because of its importance in protecting the dentition. ${ }^{16}$ Participants had nothing to eat or drink for one hour before we collected their saliva. During the saliva collection process, participants were seated comfortably in a quiet, private setting with their eyes open, and head slightly forward to provide the sample. We obtained a four-minute sample without regard to time of day, room humidity, or temperature. We instructed the participants to let the saliva flow (drool) rather than to forcefully expectorate. We weighed the saliva sample with a balance We decided to measure the samples' weight, rather than volume, because bubbles in the saliva could interfere with measuring its volume.2 On the basis of the methods and protocols used in previous studies, 2 , 9,10 we assigned a specific gravity of 1.0 to saliva, converted weight to volume and defined unstimulated hyposalivation as $0.1 \mathrm{ml} /$ minute or less.

The dental evaluator used guidelines from the National Health and Nutrition Examination Survey IV to assess the participants' oral mucosa, tongues, existing restorations, attrition levels, plaque levels, gingival recession levels and periodontal attachment levels. ${ }^{17} \mathrm{We}$ assessed the participants' mouths for the presence or absence of oral tissue dryness by determining if the oral mucosa appeared to be dry, if the lips appeared to be dry, if palpation of the salivary glands produced no saliva, and if the tongue appeared to be dry, erythematous and rough.

We used commercially available statistical software for all analyses. We presented mean and percentage distributions in the study. We used a T-test and General Linear Models (GLM) to test the mean difference between male and female participants and across age groups, respectively. We used the Pearson Correlation Coefficient to test the associations between the measures of oral tissue dryness. We determined positive predictive values (PPVs), sensitivity values and the specificity values by means of cross-tabulation analysis. The standard is based on assessment of hyposalivation, which was defined as an unstimulated salivary flow rate of $0.1 \mathrm{ml} / \mathrm{minute}$.

\section{RESULTS}

The study consisted of 252 participants. There were 164 women and 88 men (65.1 percent and 34.9 percent, respectively). The mean age of the participants was 78 years (Range: 70-94; standard deviation 5.8). Ninety-six participants (38.1 percent) had an annual household income below \$20,000, and 111 participants (44 percent) had a high school education or less.

Table 1 presents the mean unstimulated salivary flow rates and mean XI scores across age groups and sex. The average measured unstimulated salivary flow rate was $0.4 \mathrm{ml} / \mathrm{minute}$ (SD $=0.3$ ). There was a significant difference in the unstimulated flow rate between men and women $(\mathrm{t}=4.1, \mathrm{p}<0.01)$, but we found no difference across age groups $(\mathrm{F}=2.9, \mathrm{p}=0.06)$. We found no significant differences for sex, or age with the XI.

We excluded 28 participants who did not complete the XI. Twenty-seven of the participants (12.1 percent) had low unstimulated salivary flow rates of $0.1 \mathrm{ml} / \mathrm{minute}$ or less (Table 2). Eight of these participants reported having xerostomia. Of the 197 participants who had no 
hyposalivation, 35 reported having xerostomia. Among the 43 participants who reported having xerostomia, only eight had hyposalivation (PPV $=18.6$ percent)). For female participants, the sensitivity value between xerostomia and hyposalivation was 33.3 percent and the PPV was 25.8 percent. These values were higher than those of male participants, which were 0 percent for both sensitivity and PPV.

Table 3 shows the responses to the XI by participants who had hyposalivation and who reported xerostomia. Dry lips had the highest association with xerostomia. All other items on the XI were reported by fewer than 60.0 percent of the participants with xerostomia. Only about one fourth of the participants with hyposalivation (29.6 percent) also reported dry mouth.

Among 234 respondents, we clinically assessed dry mouth in 32 participants (13.7 percent) (Table 4). Of those 32 participants, 13 (5.5 percent) reported having xerostomia (sensitivity = 40.6 percent). Of the 202 participants whom we did not determine via clinical assessment to have dry mouth, 167 (71.4 percent) did not report having xerostomia (specificity $=82.7$ percent). Forty-eight participants (20.5 percent) reported having xerostomia, of whom 13 (5.6 percent) had dry mouth at clinical assessment $(\mathrm{PPV}=27.1$ percent). Of the participants whose salivary flow rate we measured, 27 had dry mouth at clinical assessment, and only eight had hyposalivation (sensitivity $=29.6$ percent); the specificity value was 90.2 percent and the sensitivity value was 28.6 percent (Table 5). We found that hyposalivation and xerostomia were not significantly related (Pearson correlation coefficient $=0.1, P=0.13$ ). Clinically assessed dry mouth correlated modestly, but significantly, with hyposalivation (Pearson correlation coefficient $=0.2 ; \mathrm{P}=0.003$ ) and self-rated xerostomia (Pearson correlation coefficient $=0.2 ; \mathrm{P}=0.010$ ).

\section{DISCUSSION}

In our study, we conducted a clinical assessment of oral dryness among older adults 70 years and older in West Virginia and determined the frequency of hyposalivation and xerostomia in this population. A total of 20.5 percent of the participants reported having xerostomia, whereas 12.1 percent had hyposalivation and 13.7 percent had clinically identified dry mouth. A modest correlation exists among these three measures of oral dryness.

The study sample was a convenience sample of older adults who volunteered to participate, rather than a more representative, probability-based sample. Therefore practitioners should use caution when generalizing these findings to older adults in the general population. Another concern is that the clinical assessment may have missed cases of hyposalivation that were transient or of a cyclic nature not evident at the time of clinical assessment of dry mouth. Medications and the diurnal cycle are known to influence the glandular secretions. Ideally, the study design should have included obtaining salivary samples taken consistently between 9 and 11 a.m. or obtaining multiple samples from each participant and averaging the measurements.

Our findings generally are consistent with those from other studies of rates of salivary gland hypofunction in middle-aged to older adults, as well as the rates of salivary gland hypofunction in the population at large. $1,9,10,18 \mathrm{We}$ found that about one-fifth (20.5 percent) of participants reported having xerostomia, which is similar to estimates from a review article indicating that xerostomia rates for older adults were between 17 and 29 percent. 1 As in other studies of older adults, the sample in our study had significantly more women than men, 65.1 percent and 34.9 percent, respectively. In a review article, the authors found that the rate of xerostomia in females is higher than that in males, 1 while in our study, we found that 22.1percent of female participants and 20.9 percent of male participants reported having xerostomia (not significantly 
different). A total of 70.4 percent of the participants in our study who had hyposalivation did not report having xerostomia.

We found that the oral dryness assessment depends on three-the clinical assessment made by an examiner, the participant's self-assessment, and the participant's unstimulated salivary flow. The results of another study showed that the sensation of xerostomia may or may not occur when people who have normal salivary flow rates because areas of localized mucosal dehydration may exist in conjunction with normal salivary flow. ${ }^{6}$ A normal salivary flow rate would not indicate an area of localized mucosal dryness. Conducting a visual assessment may be the only way to identify such localized areas. Alternatively, a low unstimulated salivary flow rate may be the only way to identify a person whose salivary glands respond quickly to the manipulation that occurs during an oral evaluation and that may mask oral dryness. It is important to respond to a person's complaint of oral dryness. Overall, each of these criteria plays a role in identifying oral dryness.

Further study is needed in the area of salivary diagnostics to improve and simplify the identification of hyposalivation, especially for older adults. Substantial impairment in salivary flow and swallowing in older adults may be the primary event leading to bacterial growth, influx of inflammatory cells, mediator-induced epithelial injury and pneumonia. ${ }^{19}$ Prevention programs for and treatment of hyposalivation and xerostomia can improve patients' quality of life $^{3}$ and oral tissue health, as well as reduce caries burden. Treatments include replacing oral fluids with water; avoiding caffeinated, sweet or acidic drinks; stimulating the salivary glands with sugarless gums and candies; using systemic sialogogic agents such as pilocarpine and cevimeline. ${ }^{6,20,21}$ In addition, an individualized prevention program which may include more frequent dental recall appointments, topical applications of fluoride gels or fluoride varnishes at home, and oral hygiene education emphasizing the importance of meticulous oral hygiene may be needed.

\section{CONCLUSIONS}

Simple and effective screening tools for xerostomia and hyposalivation would be beneficial. We have conducted our study to determine the extent to which the three measures of dry oral tissue are associated with xerostomia and hyposalivation and to use this information to identify an effective means of diagnosing xerostomia and hyposalivation. Salivary assessment is an important component of a dental evaluation. In addition to the oral examination and selfreported assessment of xerostomia, dentists may want to determine participants' unstimulated salivary flow rates periodically. In our study, the participants completed the unstimulated salivary flow rate assessment in only four minutes. Measuring unstimulated salivary flow rates could be incorporated into an oral hygiene appointment as routine care for older adults. Obtaining routine unstimulated salivary flow rates in addition to self-reported information and oral evaluations may increase early detection of oral dryness, which would assist in implementing early interventions to improve patients' quality of life.

\section{Acknowledgments}

The authors wish to acknowledge Dr. Jane Chalmers's research contributions and her dedicated efforts to advance dental care for the older adult until her untimely death in 2008. Special thanks are also extended to Elizabeth DiNapoli, Lina Bai, Valerie Ignatenko, Brian Jenson, Eric Goedereis, Ben Weinstein, William Moughamer, Stella Chapman, and Linda Brown.

This study was supported by the NIH/NIDCR grant 1R21DE016970 and the West Virginia University Health Sciences Center. 


\section{REFERENCES}

1. Guggenheimer J, Moore PA. Xerostomia: etiology, recognition and treatment. JADA 2003;134:6169. [PubMed: 12555958]

2. Valdez IH, Fox PC. Diagnosis and Management of Salivary Dysfunction. Clinical Reviews in oral Biology and Medicine 1993;4:271-277.

3. Turner M, Jahangiri L, Ship JA. Hyposalivation, xerostomia and the complete denture A systematic review. JADA 2008;139:146-150. [PubMed: 18245681]

4. Navazesh N, Satish KS, Kumar MDSc. Measuring salivary flow Challenges and opportunities. JADA 2008;139 Special Supplement:35S-40S. [PubMed: 18460678]

5. Fox PC. Differentiation of Dry Mouth Etiology. Adv Dent Res 1996;10:13-16. Available at http:// adr.iadrjournals.org/cgi/reprint/10/1/13 access verified 7/7/2008. [PubMed: 8934917]

6. Narhi TO. Prevalence of Subjective Feelings of Dry Mouth in the Elderly. J Dent Res 1994;73(1):2025. [PubMed: 8294614]

7. Jones JA, Kressin NR, Spiro A, Randall CW, et al. Self-reported and Clinical Oral Health in Users of VA Health Care. Journal of Gerontology: Medical Sciences 2001;56A:M55-M62.

8. Hay EM, Thomas E, Pal B, Hajeer A, Chambers H, Silman AJ. Weak association between subjective symptoms of and objective testing for dry eyes and dry mouth: results from a population based study. Ann Rheum Dis 1998;57:27-24.

9. Dawes C. Salivary flow patterns and the health of hard and soft tissues. JADA 2008;139 Special Supplement:18S-24S. [PubMed: 18460676]

10. Navazesh M. How can oral health care providers determine if patients have dry mouth? JADA 2003;134(5):613-618. [PubMed: 12785497]

11. Caplan DJ, Hunt RJ. Salivary flow and risk of tooth loss in an elderly population. Community Dent Oral Epidemiol 1996;24:68-71. [PubMed: 8833518]

12. Wu B, Plassman BL, Liang J, Wei L. Cognitive Function and Dental Care Utilization Among Community-Dwelling Older Adults. American Journal of Public Health 2007;97:2216-2221. [PubMed: 17971546]

13. Reisine ST, Bailit HL. Clinical Health Status and Adult Perceptions of Oral Health. Soc Sci Med Med Psychol Med Sociol 1980;14A:597-605. [PubMed: 7209633]

14. Thomson WM, Chalmers JM, Spencer AJ, Williams SM. The Xerostomia Inventory: A multi-item approach to measuring dry mouth. Community Dent Health 1999;16(1):12-17. [PubMed: 10697349]

15. Wu B, Goedereis E, Crout R, Plassman BL, DiNapoli E, McNeil DW, Wiener MA, Boone M, Wiener RC, Kao EC, Bai L. Recruitment of Rural and Cognitively Impaired Elders for Dental Research. Special Care in Dentistry. (in press).

16. Thomson WM, Chalmers JM, Spencer AJ, Ketabi M. The occurrence of xerostomia and salivary gland hypofunction in a population-based sample of older South Australians. Special Care in Dentisry 1999;19:20-23.

17. National Center for Health Statistics. National Health and Nutrition Examination Survey: DENTAL EXAMINERS PROCEDURES MANUAL. 2001 Jan [Accessed 11/14/209]. Revised http://www.cdc.gov/nchs/data/nhanes/oh-e.pdf

18. Thomson WM, Williams SM. Further testing the xerostomia inventory. Oral Surg Oral Med Oral Pathol Oral Radiol Endod 2000;89:46-50. [PubMed: 10630941]

19. Palmer LB, Albulak K, Fields S, Filkin AM, Simon S, Smaldone GC. Oral Clearance and Pathogenic Oropharyngeal Colonization in the Elderly. Am J Respir Crit Care Med. 2001 164:464-468. Available at http://ajrccm.atsjournals.org/cgi/content/full/164/3/464. [PubMed: 11500351]

20. Stookey G. The effect of saliva on dental caries. JADA 2008;139 Special Supplement:6S-8S. [PubMed: 18460674]

21. Curro FA. Gum chewing as an adjunct to use of medications. JADA 2008;139 Special Supplement: 6S-8S. [PubMed: 18460674] 
Table 1

Mean Unstimulated Salivary Flow Rates and Mean Xerostomia Inventory Scores according to by Sex and Age Group

\begin{tabular}{|l|l|l|}
\hline & $\begin{array}{l}\text { Mean Unstimulated Salivary } \\
\text { Flow Rates in ml/min(SD) }\end{array}$ & $\begin{array}{l}\text { Mean Xerostomia } \\
\text { Inventory (SD) }\end{array}$ \\
\hline Overall & $0.4(0.3)$ & $21.7(7.4)$ \\
\hline $\begin{array}{l}\text { Sex } \\
\text { Women }\end{array}$ & $0.3(0.3)$ & $22.1(7.4)$ \\
\hline Men & $0.5(0.4)$ & $20.9(7.5)$ \\
\hline $\begin{array}{l}\text { Age } \\
<75\end{array}$ & $0.4(0.3)$ & $21.0(7.5)$ \\
\hline $75-84$ & $0.4(0.4)$ & $21.8(7.0)$ \\
\hline$>85$ & $0.3(0.2)$ & $22.9(8.5)$ \\
\hline
\end{tabular}

There was significant difference in the unstimulated flow rate across gender groups $(\mathrm{t}=4.12, \mathrm{p}<0.001)$, but no difference across age group $(\mathrm{F}=2.92$, $\mathrm{p}=0.056$ ). No differences were found for the Xerostomia Inventory at the 0.05 level. 
Table 2

Relationship of Hyposalivation $^{1}$ and Xerostomia ${ }^{2}$

\begin{tabular}{ccccc}
\hline & \multicolumn{3}{c}{ Hyposalivation } \\
& & Yes & No & Total \\
\hline All participants & & & & \\
Xerostomia & Yes & $8(3.6 \%)$ & $35(15.6 \%)$ & $43(19.2 \%)$ \\
& No & $19(8.5 \%)$ & $162(72.3 \%)$ & $181(80.8 \%)$ \\
& Total & $27(12.1 \%)$ & $197(87.9 \%)$ & $224(100.0 \%)$ \\
Women & & & & \\
Xerostomia & Yes & $8(5.5 \%)$ & $23(15.8 \%)$ & $31(21.2 \%)$ \\
& No & $16(11.0 \%)$ & $99(67.8 \%)$ & $115(78.8 \%)$ \\
Men & Total & $24(16,4 \%)$ & $122(83.6 \%)$ & $146(100.0 \%)$ \\
Xerostomia & & & & \\
& Yes & $0(0.0 \%)$ & $12(15.4 \%)$ & $12(15.4 \%)$ \\
& No & $3(3.8 \%)$ & $63(80.8 \%)$ & $66(84.6 \%)$ \\
& Total & $3(3.8 \%)$ & $75(96.2 \%)$ & $78(100.0 \%)$ \\
\hline
\end{tabular}

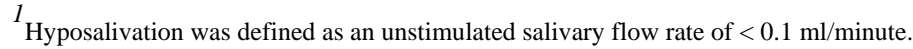

2 Participants could respond "never," "hardly ever," “occasionally," "fairly often," or "very often." The associated values were 1 through 5 respectively. Affirmative answers were defined as "fairly often," or "very often." 


\section{Table 3}

Affirmative Responses to the Xerostomia Inventory by those with hyposalivation and those reporting xerostomia

\begin{tabular}{|l|c|c|}
\hline Participants reporting affirmative responses ${ }^{\mathbf{I}}$ to: & $\begin{array}{c}\text { Participants with } \\
\text { Hyposalivation } \mathbf{I} \\
\mathbf{N}=\mathbf{2 8}\end{array}$ & $\begin{array}{c}\text { Participants reporting } \\
\text { Xerostomia } \\
\mathbf{N}=\mathbf{4 3}\end{array}$ \\
\hline Swallowing difficulty—certain foods & $1(3.6 \%)$ & $2(4.7 \%)$ \\
\hline Eating difficulty—dry foods & $3(11.1 \%)$ & $7(16.7 \%)$ \\
\hline Eating-associated dry mouth & $2(7.1 \%)$ & $5(11.6 \%)$ \\
\hline Dry nose & $4(14.3 \%)$ & $17(39.5 \%)$ \\
\hline Dry face & $4(14.3 \%)$ & $11(25.6 \%)$ \\
\hline Eating hard candy/cough drops to relieve dry mouth & $4(14.3 \%)$ & $9(20.9 \%)$ \\
\hline Sleep interruptions to drink & $3(10.7 \%)$ & $14(32.6 \%)$ \\
\hline Dry eyes & $11(39.3 \%)$ & $19(44.2 \%)$ \\
\hline Dry lips & $8(29.6 \%)$ & $26(60.5 \%)$ \\
\hline Dry mouth & $8(29.6 \%)$ & $43(100.0 \%)$ \\
\hline Swallowing aided with liquid & $4(14.4 \%)$ & $14(32.6 \%)$ \\
\hline
\end{tabular}

${ }^{1}$ Hyposalivation was defined as an unstimulated salivary flow rate of $<0.1 \mathrm{ml} /$ minute.

2 Participants could respond "never," "hardly ever," “occasionally," "fairly often,”or "very often.” The associated values were 1 through 5 respectively. Affirmative answers were defined as "fairly often," or "very often." 
Table 4

Relationship of Clinically Assessed Dry Mouth and self-reported xerostomia

\begin{tabular}{|l|r|r|r|}
\hline \multirow{2}{*}{} & \multicolumn{3}{|c|}{ Clinically Assessed Dry Mouth (N \%) } \\
\cline { 2 - 4 } & Yes & No & Total \\
\hline $\begin{array}{l}\text { Self-reported } \\
\text { Xerostomia }\end{array}$ & & & \\
\hline Yes & $13(5.6 \%)$ & $35(15.0 \%)$ & $48(20.5 \%)$ \\
\hline No & $19(8.1 \%)$ & $167(71.4 \%)$ & $186(79.5 \%)$ \\
\hline Total $^{l}$ & $32(13.7 \%)$ & $202(86.3 \%)$ & $234(100.0 \%)$ \\
\hline & & & \\
\hline
\end{tabular}

Note:

${ }^{l}$ Percentages total across rows. Sample sizes varied due to missing cases. 
Table 5

Relationship of Clinically Assessed Dry Mouth and Hyposalivation

\begin{tabular}{|l|r|r|r|}
\hline \multirow{2}{*}{} & \multicolumn{3}{|c|}{ Clinically Assessed Dry Mouth (N \%) } \\
\cline { 2 - 4 } & Yes & No & Total \\
\hline Hyposalivation & & & \\
\hline Yes & $8(3.5 \%)$ & $20(8.7 \%)$ & $28(12.1 \%)$ \\
\hline No & $19(8.2 \%)$ & $184(79.7 \%)$ & $203(87.9 \%)$ \\
\hline Total $^{l}$ & $27(11.7 \%)$ & $204(88.3 \%)$ & $231(100.0 \%)$ \\
\hline
\end{tabular}

Note:

${ }^{1}$ Percentages total across rows. Sample sizes varied due to missing cases. 